\title{
CORRIGENDUM
}

\section{Nutrition labelling, marketing techniques, nutrition claims and health claims on chip and biscuit packages from sixteen countries - Corrigendum}

\author{
Alexandra J Mayhew, Karen Lock, Roya Kelishadi, Sumathi Swaminathan, \\ Claudia S Marcilio, Romaina labal, Mahshid Dehghan, Salim Yusuf and Clara K Chow
}

First published online 3 July 2015

doi:10.1017/S1368980015000658. Published online by Cambridge University Press 30 March 2015

Author affiliation 3, Isfahan Cardiovascular Research Center, Isfahan University of Medical Sciences, Isfahan, Islamic Republic of Iran

Should have read:

Isfahan Cardiovascular Research Center, Isfahan Cardiovascular Research Institute, Isfahan University of Medical Sciences, Isfahan, Iran.

\section{Reference}

Mayhew AJ, Lock K, Kelishadi R, Swaminathan S, Marcilio CS, Iqbal R, Dehghan M \& Chow CK (2015) Nutrition labelling, marketing techniques, nutrition claims and health claims on chip and biscuit packages from sixteen countries. Public Health Nutrition. Published online by Cambridge University Press 30 March 2015, doi:10.1017/S1368980015000658. 